(survanta $4-8 \mathrm{ml} / \mathrm{kg}$ ) are used in our babies. We performed a subgroup analysis comparing the MIST success rate and the relevant clinical outcomes in our prospective observational cohort of MIST babies (14 times in 13 babies) that received porcine surfactant $(n=9$, one time each in nine babies) versus those that received bovine surfactant $(n=5$, one time each in three babies and two times in 1 baby).

Results Nine babies (gestation 27 - 36 weeks and birth weight $0.95 \mathrm{~kg}$ to $2.4 \mathrm{~kg}$ ) received Curosurf $(1.5 \mathrm{ml} / \mathrm{kg}, 200 \mathrm{mg} / \mathrm{kg}$ ) by MIST one time in each baby. The median age of administration of Curosurf was $12.5 \mathrm{~h}$. Conduits used for MIST were by infant feeding tube in eight babies and LISA (Less Invasive Surfactant Administration) catheter in one baby. Four babies (gestation 27 - 34 weeks and birth weight $1.04 \mathrm{~kg}$ to 2.81 $\mathrm{kg}$ ) received Survanta $(4-8 \mathrm{ml} / \mathrm{kg}, 100-200 \mathrm{mg} / \mathrm{kg})$ five times by MIST one time each in three babies and two times in one baby. The median age of administration of survanta was $13 \mathrm{~h}$. The conduits used for the MIST were infant feeding tube $\mathrm{s}$ on two occasions, LISA catheter once and $2.0 \mathrm{~mm}$ Endotracheal Tube with surfactant filled syringe directly attached to the syringe hub on two occasions in one baby. Syringe air flush and checking for surfactant reflux was done as per MIST procedure protocol on all occasions after the instillation of surfactant. Success rate of MIST procedure was $100 \%$ irrespective of surfactant preparation. Equally beneficial clinical outcomes are seen in babies receiving bovine surfactant MIST and porcine surfactant MIST.

Conclusions Surfactant reflux is nullified with the incorporation of syringe air flush technique in MIST thereby ensuring equally better clinical outcomes with higher volume bovine surfactant versus porcine surfactant in this cohort. A higher dose volume surfactant can be effectively delivered by MIST.

\section{PATIENT PERSPECTIVES REGARDING ONLINE CONSULTATIONS DURING THE COVID-19 PANDEMIC}

John Cheri Mathews, Deepa Elizabeth Mathew, Shipra Mathur, Natasha Susan John, Suneela Nayak, Vinay Bothra. India

\subsection{6/bmjpo-2021-RCPCH.229}

Background Introduction: COVID-19 and the associated lockdown has prevented patients from accessing healthcare professionals through the traditional face-to-face clinic visits. Modes of consultation have therefore adapted to overcome this barrier; virtual or online consultations have become a popular alternative for people with access to technology. The literature on patient perspectives about this mechanism is still evolving.

Objectives Aim: To explore patient perceptions regarding virtual consultations

Methods A voluntary online survey instrument using a mix of quantitative and qualitative questions was administered to patients across 3 major cities using a social media platform, WhatsApp. The aim was to explore the characteristics of users, perceived advantages and disadvantages of online consultations and patient satisfaction.

Results There were 461 respondents (M 51.4\%: F 48.6\%) that had consulted doctors online. $91 \%$ of them lived in 8 major metro cities. Interestingly, over $80 \%$ respondents had never sought online consultation before the COVID-19 pandemic. $52 \%$ patients accessed multiple (2-3) consultations in the 10 months since the start of the lock-down in March 2020. While $62 \%$ consulted their regular doctor, $19 \%$ accepted recommendations from friends and family and 10\% used online platforms. $45 \%$ of consultations were via videocalls, 28\% through WhatsApp and 20\% via telephone calls. Prescriptions were provided via WhatsApp in $41 \%$ cases, online portals in 32\%, email in $13 \%$ and a photograph of handwritten prescription in $13 \%$ cases. The vast majority (90\%) felt that the time provided by the doctor was adequate. $55 \%$ of patients paid via $G$ pay while $28 \%$ were prepaid through online portals. There were no audio or video connectivity issues in $90 \%$ cases. $13 \%$ patients had to go for a faceto-face consultation within 7 days of the online consultation as the clinical problem had not been resolved adequately. Patients felt that the main advantages of online consultations included a lower risk of infection (77\%), reduced waiting time $(57 \%)$ and travel time (58\%). The main disadvantages cited included a lack of physical examination (73\%), a perception that this was not as satisfying as a face-to-face consultation (36\%), inability to adequately communicate their problem (24\%) and an inability to show past reports (13\%). $78 \%$ patients rated their online consultations as either a $4 / 5$ or $5 / 5$ satisfaction level. Given the choice after the pandemic, almost two-thirds (64\%) felt they would still prefer face-face consultations.

Conclusions For the vast majority of patients (80\%), this pandemic provided the first ever opportunity to choose a virtual platform to seek clinical care. The high level of satisfaction for online consultations suggests that this mechanism of patient-provider clinical service provision might well be an increasingly popular intervention after restrictions are lifted. As digital penetration improves, weaknesses identified in the current system of online consultations (e.g., inability to conduct physical examinations and inadequate communication) could be mitigated through evolving technologies like digital stethoscopes and better communication tools. It brings into focus the need for regulations to keep pace with this rapidly evolving trend to ensure that the virtual patient-provider interaction remains a safe, secure and confidential way to access clinical services.

\section{RESPIRATORY VIRUS INFECTION-ASSOCIATED MORTALITY AMONG CRITICALLY ILL CHILDREN: A RETROSPECTIVE SINGLE-CENTRE COHORT STUDY}

Divyapoorani Ravichandran, Kian Boon, Joel Lim, Poh Hui Wee, Chee Fu Yung, Jan Hau Lee, Kee Thai Yeo. Singapore

\subsection{6/bmjpo-2021-RCPCH.230}

Background Limited data from Singapore indicates that respiratory virus infection (RVI) burden is high and comparable to other settings in temperate countries. Acute lower respiratory infections from RVIs are important contributors to mortality in children $<5$ years of age, with most deaths occurring in the first year of life.

Objectives This study aims to describe the burden and epidemiology of RVI-associated mortality among children admitted to the paediatric intensive care unit (PICU).

Methods We performed a linkage analysis of PICU mortality database with laboratory confirmed RVI database of patients $<18$ years old from 2010 to 2019. RVI-associated mortality was defined as cases with a laboratory confirmed RVI from any respiratory sample within 14 days prior to the certified date of death. 
Results Over the 10-year period, there were 339 (5.6\%) deaths out of 6101 admissions to the PICU. 67 (19.8\%) out of a total of 339 deaths were associated with RVIs. Patients with RVI-associated mortality had a median age of 3 years (Q1 - Q3, 0 - 8). The majority were male $(n=38,56.7 \%) .23$ $(34.3 \%)$ of the patients were born preterm and $42(62.7 \%)$ had co-morbid conditions. Influenza (22.7\%), adenovirus (17.3\%), respiratory syncytial virus (RSV) (16\%) and rhinovirus $(16 \%)$ were the most common viruses isolated. Eight patients (11.9\%) had RVI coinfection. The most common documented cause of death in this cohort of RVI-associated mortalities was viral and/or secondary bacterial infections (76.1\%) followed by cardiovascular causes (7.5\%). The median hospital length of stay prior to death was 8 days (Q1 - Q3, 3 - 15).

Conclusions The burden of RVI-associated mortality is high among critically ill children. These data on the burden and age-specific distribution of RVI-associated mortality in children are critical in informing infection prevention practices among high-risk groups and immunization public health policies for RVIs. Efforts to improve influenza vaccination coverage especially in children with comorbidities or history of prematurity could have a significant impact in reducing this burden.

\section{SCOPING REVIEW ON THE EFFECTIVENESS OF THE USE OF TECHNOLOGY FOR PSYCHOSOCIAL SCREENING OF ADOLESCENTS IN URBAN HEALTH CARE SETTINGS}

Rebecca Loh. Australia

\subsection{6/bmjpo-2021-RCPCH.231}

Background The number of adolescents presenting with psychosocial issues at urban hospital pediatric emergency departments have increased significantly since 2000. Early psychological intervention has been proven to prevent future disabilities. Consequences from missing or delayed diagnosis of psychosocial issues in adolescence include increased suicide incidences and potential harm from extensive and invasive investigations. However, there is limited time and resources to evaluate youth for psychosocial stressors in healthcare facilities (Emergency department, clinics). Commonly, medical concerns are addressed first. Furthermore, youths are reluctant to disclose threats to their mental and social well-being when reviewed.

Objectives The effectiveness of technology in psychosocial screening of adolescents in urban health care settings was examined. Specifically, the rate and scope of detection of psychosocial stressors. This would allow for better understanding on how mental health screening resources for adolescence should be allocated. Advancements in psychosocial technological screening can potentially prevent unnecessary consumption of healthcare resources.

Methods A scoping review was used to study scientific literature regarding screening methods for mental health disorders in adolescence. Arksey and O'Malley framework was used to review articles from electronic databases such as BMJ, Cochrane, EBM, Pubmed, Uptodate. The review included cohort and experimental studies of adolescents (12-18 years old) from urban areas who were screened for mental health disorders using different screening tools. A secondary reviewer was asked to do further analysist of the articles during the second round of screening of the articles. Analytical research methods were then used to compare the detection rates and time taken to detect mental health problems in adolescence, using the various screening tools, both online and face to face.

Results 113 search articles were identified. 79 articles were yielded to be utilised in the review. Journal articles were included in the review if they were in English, published after 2000, conducted in metropolitan areas. Youths included in the studies were those who sought help at healthcare facilities (bothemergency department or clinic) in densely populated cities with easy access to both healthcare facilities and technology. Screening tools studied included standalone screening specific softwares, internet based softwares, telephone applications. Most screening tools utilised questionnaires and 3 utilised games. Journal articles studied included 37 randomized controlled trials, 14 Meta-Analysis, 7 Systematic Review, 2 Cohort Studies.

59 articles reflected that online psychosocial tools reached a wider spectrum of youths. 43 indicated that number of youths willing to undergo screening increased when online psychosocial tests were utilised. Screening rates for psychosocial problems of the target population of adolescence increased $6 \%$, compared with traditional face-to-face interview methods. Furthermore, youths were more willing to share openly on an online platform according to 5 research articles. As such, identification of mental disorders in youths was faster and more accurate when an online platform was utilised. Because of that, disclosure rate of the psychosocial stressors increased by $17 \%$ when online screening tools were utilised.

Conclusions It can be concluded that online screening tools are more effective, with regards to speed and scope of detection of mental health disorders amongst adolescence living in urban areas.

\section{RISK FACTOR AND OUTCOME OF ACUTE KIDNEY INJURY AMONG CRITICALLY ILL CHILDREN}

Wun Fung Hui, Vivian Pui Ying Chan, Kam Lun Hon, Man Hong Poon. Hong Kong

\subsection{6/bmjpo-2021-RCPCH.232}

Background Acute kidney injury (AKI) is an independent predictor of morbidity and mortality among critically ill children. However, epidemiological data in Asian paediatric populations remain scarce.

Objectives We presented the result of the interim analysis of an ongoing prospective cohort study on the epidemiology of AKI and electrolytes disturbances and their potential relationships with nephrotoxic medications (E-AKI-Drug) in a newly established paediatric intensive care unit (PICU).

Methods We enrolled all children aged 1 month to 18 years old admitted to the PICU of our hospital after June 2020. Those with pre-existing chronic kidney disease, impaired renal function for $\geq 3$ months, immediate post-renal transplant and short stay in PICU $<1$ day with no blood taking would be excluded. Children without a urinary catheter would be excluded from urine calculation. AKI would be defined using the KDIGO criteria. The medication records from 14 days prior to PICU admission to PICU discharge would be retrieved and reviewed by an independent pharmacist. The results of the initial 4 months of data collected would be presented. 